\title{
El riñón del niño prematuro: riesgos a largo plazo
}

\section{The kidney of the premature child: long-term risks}

\section{Felipe Cavagnaro SM ${ }^{\mathrm{a}}$}

aDepartamento de Pediatría, Clínica Alemana de Santiago. Facultad de Medicina, Clínica Alemana - Universidad del Desarrollo. Santiago, Chile

Recibido: 13 de enero de 2020; Aceptado: 8 de marzo de 2020

\section{¿Qué se sabe del tema que trata este estudio?}

El desarrollo anatómico y funcional de nuestros órganos puede verse alterado cuando el nacimiento ocurre en forma prematura. Con relación a esta situación, el riñón humano puede no lograr desarrollar una masa nefronal completa si nace muy prematuro, y por ende más susceptible de daño renal crónico.

\section{¿Qué aporta este estudio a lo ya conocido?}

La evidencia científica actual demuestra que el riñón del niño prematuro es más propenso a desarrollar un daño crónico a futuro, así como hipertensión arterial como coadyuvante de riesgo cardiovascular. Estos hechos hacen necesario un seguimiento de largo plazo de función renal y presión arterial en este grupo de niños/as.

\section{Resumen}

El recién nacido prematuro se enfrenta a las condiciones extrauterinas con sistemas aún inmaduros, tanto anatómica como fisiológicamente. El riñón termina de desarrollarse a finales del tercer trimestre del embarazo, por lo que está especialmente expuesto a alterar su desarrollo normal en caso de nacer en forma prematura. Esta situación puede condicionar, entre otras consecuencias, una menor masa renal funcional y cambios microvasculares que representan un riesgo elevado de hipertensión arterial y daño renal crónico en el largo plazo. En el presente artículo se analiza la evidencia existente actual sobre estos riesgos en los prematuros y se ofrece un esquema de seguimiento de estos niños desde el punto de vista nefrológico.

\begin{abstract}
The premature newborn faces extrauterine conditions with some systems still immature, both anatomically and physiologically. The kidney finishes developing at the end of the third trimester of pregnancy, so it is especially exposed to alter its normal development if preterm birth occurs. This situation may condition, among other consequences, a lower functional renal mass and microvascular changes comprising a high risk of chronic kidney disease in the long term and arterial hypertension. This article analyzes the current evidence on these risks in premature infants and offers a nephrology follow-up scheme of these children.
\end{abstract}

\section{Palabras clave:}

Prematuridad; recién nacido; bajo peso de nacimiento; función renal; hipertensión arterial; enfermedad renal crónica

\section{Keywords:}

Prematurity; newborn; low birth weight; renal function; arterial hypertension; chronic kidney disease 


\section{Introducción}

El nacimiento prematuro, definido como aquel que ocurre antes de las 37 semanas de edad gestacional (EG) es un evento desafortunadamente frecuente. La incidencia global de partos prematuros es de aproximadamente el $10 \%$, siendo más alta en países en vías de desarrollo ${ }^{1-3}$. En Chile esta cifra alcanza al $7 \%{ }^{3}$. Las causas de partos prematuros en países de altos ingresos se relacionan a avanzada edad materna, obesidad materna, reproducción asistida, gestaciones múltiples y enfermedades crónicas en la gestante, en cambio, en países de bajos ingresos la maternidad en adolescentes, desnutrición materna, preeclampsia, infecciones maternas y pobreza son las causas más frecuentes ${ }^{1}$. La prematurez, a su vez, es la principal causa de mortalidad neonatal, especialmente en países de bajos ingresos ${ }^{4}, \mathrm{y}$ un importante factor de discapacidad futura ${ }^{1}$.

Si bien inicialmente los esfuerzos médicos en este grupo de recién nacidos (RN) estaba enfocado en aumentar su supervivencia, el avance del desarrollo tecnológico y del conocimiento de la fisiología fetal nos han permitido preocuparnos de lograr una sobrevida sin secuelas funcionales derivadas de la propia inmadurez o de las terapias empleadas. Los conocimientos actuales nos han mostrado que el desarrollo madurativo de algunos sistemas, tanto anatómico como funcional, puede completarse en las últimas semanas del embarazo de término, como ocurre con el riñón ${ }^{5,6}$. En condiciones de prematurez, esta madurez morfo-funcional del tejido renal puede no ocurrir y, teóricamente, puede derivar en una situación fisiopatológica que genere un daño acumulativo que se exprese en etapas posteriores de la vida. El presente artículo explora la evidencia científica actual relacionada a los posibles efectos futuros sobre el riñón y su función, en el contexto de haber nacido prematuro y/o con bajo peso de nacimiento (BPN).

\section{Desarrollo renal prenatal y post natal precoz}

La nefrogénesis, y específicamente la glomerulogénesis, ocurren intrauterinamente desde la semana 9 hasta la semana 34 a 36 de EG, con más del $60 \%$ de los nefrones siendo formados en el último trimestre del embarazo $^{2,6}$. Este desarrollo sigue un sentido centrífugo, en el que los glomérulos más nuevos van apareciendo en las capas más externas de la corteza y los más antiguos van quedando en regiones más profundas. La producción de orina fetal se inicia a las 9-10 semanas de EG y formará un significativo componente del volumen de líquido amniótico ${ }^{7,8}$. Debido a la alta resistencia vascular fetal intrauterina, el flujo sanguíneo renal y, por ende, la velocidad de filtración glomerular
(VFG), es baja ${ }^{8}$. En niños de término, el número de nefrones al nacer es muy variable, y va de 210.000 hasta 2,7 millones, un número muy relacionado al peso de nacimiento y a la $\mathrm{EG}^{9}$. La dotación o masa nefronal que se alcance en ese momento será con la que un individuo afronta el resto de su vida. Una excepción a esto ocurre en RN muy prematuros, en los que la nefrogénesis puede continuar en la vida extrauterina por 40 días más, siempre que el ambiente que rodea al niño y su situación clínica sean óptimos, lo que incluye buena hemodinamia y oxigenación, nutrición adecuada y evitar nefrotóxicos ${ }^{10}$. Algunos de estos nuevos nefrones son anormales y envejecen en forma acelerada ${ }^{7,11}$. Si el RN prematuro sobrevive con una disminución marcada del número de nefrones por la detención de la nefrogénesis intra o extra útero, se aplica el concepto de "oligonefropatía del prematuro" congénita o adquirida, respectivamente ${ }^{2,8}$. Esto puede conducir a riñones de menor tamaño aunque generalmente con igual velocidad de filtración glomerular (VFG) que un niño de término de igual edad, debido a un estado de hiperfiltración de cada glomérulo remanente. Para lograr esta hiperfiltración, los glomérulos usualmente aumentan de tamaño (glomerulomegalia) ${ }^{11,12}$.

\section{Riesgos a largo plazo}

En el análisis de complicaciones renales a largo plazo es importante recalcar que la mayoría de los estudios al respecto adolecen de tener un período de seguimiento relativamente corto, debido especialmente a que la mayor sobrevida de RN muy prematuros, población de alto riesgo de secuelas, es una realidad de décadas recientes ${ }^{1,2,9,13}$. Las complicaciones renales agudas, mejor estudiadas, se relacionan principalmente a inmadurez en la función tubular (tubulopatía del prematuro) y comprenden problemas relacionados a inadecuado manejo del agua libre, desbalance electrolítico y ácido-base, y pérdidas excesivas de minerales y proteínas $^{14}$.

De las posibles complicaciones a largo plazo es importante destacar (figura 1):

\section{a. Hipertensión arterial}

La asociación entre RN prematuro y presión arterial (PA) más elevada parece estar bien establecida, aunque no claramente como hipertensión arterial (HTA), para lo cual podría requerirse más tiempo de seguimiento ${ }^{1,2,13,15}$. En el caso de adolescentes y adultos jóvenes nacidos prematuramente varios estudios han observado mayores tasas de PA elevada e HTA, e inversamente proporcional a la prematurez del nacimiento $^{16,17}$. En un pequeño estudio local en escolares nacidos muy prematuramente (menores de 32 sema- 
Figura 1. Esquema de la progresión del daño renal en prematuros. BPN: bajo peso de nacimiento, UCIN: Unidad de Cuidados Intensivos Neonatales.

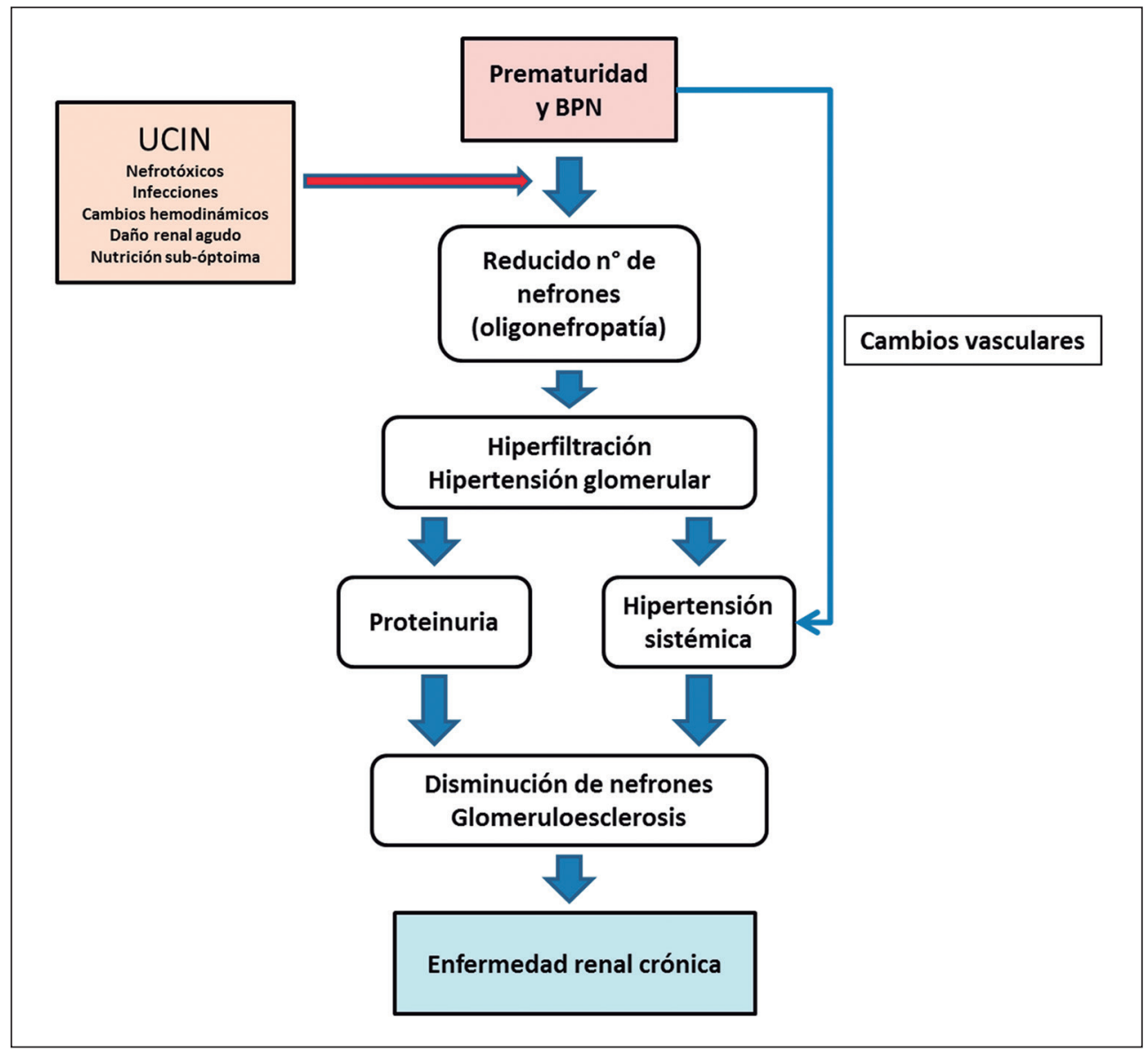

nas EG o menos de $1.500 \mathrm{~g}$ al nacer), el registro de monitoreo ambulatorio de PA (MAPA) demostró una alta prevalencia de alteraciones, principalmente la ausencia de diferencia de PA día vs noche, lo que se relaciona con mayor riesgo de progresión a $\mathrm{HTA}^{18}$.

Un estudio de metaanálisis reciente encontró que las PA sistólica y diastólica fueron en promedio 3,4 mmHg y 2,1 mmHg más elevadas, respectivamente, en adultos jóvenes nacidos en forma prematura, comparado con sus pares nacidos a término ${ }^{16}$. Otro estudio similar cifró esta diferencia en 4,2 $\mathrm{mmHg}$ para la PA sistólica y 2,6 mmHg para la PA diastólica ${ }^{19}$. Un tercer estudio evaluó el efecto futuro de ser RN pequeño (PEG) vs adecuado (AEG) para la EG en relación a cifras tensionales en adultos, mostrando un PA sistólica $7,2 \mathrm{mmHg}$ mayor en los primeros ${ }^{20}$. Si bien estas diferencias parecen no ser importantes, es claro que conllevan un mayor riesgo cardiovascular; en una población general se calcula que $4 \mathrm{mmHg}$ de diferencia de PA está asociada a una reducción de $20 \%$ en la mortalidad por accidentes vasculares encefálicos y a un 15\% de menor mortalidad de otras enfermedades vasculares, especialmente en individuos más jóvenes ${ }^{21}$. Interesantemente, mujeres nacidas muy precozmente $(<32$ semanas EG) tienen mayor frecuencia de complicaciones del embarazo, con un 50\% de aumento en HTA gestacional, pre eclampsia y, aún, HTA crónica ${ }^{13,15,22}$. A su vez, debido a la preeclampsia, hay más partos prematuros, y estos tienen mayor riesgo de PA elevada, cerrando de esta manera un círculo vicioso ${ }^{13,15,23}$. Las posibles causas para este mayor riesgo de HTA en RN prematuros son múltiples, destacando una mayor rigidez de las paredes vasculares por baja densidad de elastina, diámetro aórtico reducido, reducida densidad capilar asociado a un perfil molecular anti-angiogénico, alteraciones vasculares por daño endotelial oxidativo y envejecimiento vascular precoz, mayor sensibilidad al sodio o menor excreción de éste, y factores maternos/gestacionales que condicionan una epigenética de riesgo hipertensógeno ${ }^{5,13,15,24}$. Un exagerado aumento del índice de masa corporal post natal en prematuros también puede llevar a un aumento del riesgo de HTA futura ${ }^{1,4}$.

\section{b. Riesgo de daño renal crónico}

La prematuridad extrema, especialmente cuando se asocia a un período postnatal inmediato con patologías 
graves (ej.: enterocolitis necrotizante, insuficiencia respiratoria) induce a una oligonefropatía del prematuro, a lo cual pueden agregarse otro tipo de factores que aumenten el daño renal futuro como asfixia neonatal, drogas nefrotóxicas, sepsis y nefrocalcinosis ${ }^{1,2,13}$. De este modo, y de acuerdo a la hipótesis de Brenner, los cambios hemodinámicos adaptativos en un número reducido de glomérulos producen una hipertensión glomerular, glomerulomegalia y finalmente glomeruloesclerosis $^{25}$. Un metaanálisis, que incluyó más de 2 millones de individuos de 31 estudios, encontró que el bajo peso de nacimiento estuvo asociado con aproximadamente un $80 \%$ de aumento de probabilidad de albuminuria, un $80 \%$ de disminución sostenida de la VFG y un $60 \%$ de mayor probabilidad de daño renal crónico terminal en etapas posteriores de la vida, cuando comparado con individuos nacidos con peso normal ${ }^{26}$. Otro estudio noruego que analizó esta asociación en el registro clínicos de un gran número de individuos concluyó que existe un riesgo relativo de 1,78 de desarrollar falla renal crónica terminal en todos los niños con peso de nacimiento menor que el percentil $10^{27}$. Un reciente estudio de caso-control en niños japoneses con desarrollo de daño renal crónico durante la niñez encontró que un 21\% de los casos eran atribuibles a BPN y existió una fuerte correlación entre prematurez y daño renal crónico ${ }^{28}$. Un elemento confundente en muchos de estos análisis es la falta de definición del concepto "Bajo peso de nacimiento", en el cual podemos tener RN prematuros con bajo o adecuado peso para la edad gestacional, así como $\mathrm{RN}$ de término pero PEG (retraso de crecimiento intrauterino). Hay evidencia publicada en relación que, a igual prematurez al nacer, la VFG y la reserva funcional renal en etapas posteriores es más baja en los nacidos PEG vs $\mathrm{AEG}^{1}$. Otro factor que también puede alterar esta asociación es la forma en que estos prematuros sobrevivieron a sus primeros meses de vida, incluyendo patologías graves, uso de nefrotóxicos e inadecuada nutrición postnatal.

\section{c. Otros riesgos}

Es probable que un paciente con sospecha de oligonefropatía del prematuro esté más expuesto a desarrollar una progresión más rápida hacia el daño renal crónico si es afectado por impactos adicionales como insuficiencia renal aguda, glomerulonefritis o condiciones que conlleven riesgo de daño renal como diabetes, obesidad o hipertensión arterial ${ }^{13,29}$. Entre las patología estudiadas que progresan más rápidamente si el paciente tuvo un bajo peso de nacimiento están la nefropatía por IgA, la nefropatía membranosa, la enfermedad por cambios mínimos, la glomeruloesclerosis focal y segmentaria, la enfermedad renal poliquística autosómica dominante y el síndrome de Alport ${ }^{13}$. En niños con síndrome nefrótico idiopático, un estu- dio mostró un peor daño histológico en aquellos niños nacidos con bajo peso de nacimiento ${ }^{30}$. De hecho, se encontró un mayor porcentaje de glomeruloesclerosis focal y segmentaria córtico resistente en este grupo; y de los niños córtico sensibles, aquellos con BPN tenían un curso de enfermedad más severa, caracterizado por córtico dependencia y uso más frecuente de drogas inmunosupresoras adicionales. Estos datos sugieren un rol condicionante de cambios hemodinámicos y podocitarios debido a una reducida masa nefronal en $\mathrm{BPN}^{30}$.

Otra complicación renal frecuente en los prematuros es el desarrollo de nefrocalcinosis, sugiriendo una maduración diferenciada de los túbulos renales expresando hipercalciuria e hipocitraturia ${ }^{2}$ y un lento flujo de orina que facilitaría el depósito de cristales de calcio, riesgo que aumenta por el uso de furosemida, aminoglicósidos, corticoides y nutrición parenteral ${ }^{1}$.

\section{Seguimiento nefrológico de recién nacidos prematuros}

Los pediatras que hacen el seguimiento clínico de estos niños, especialmente los muy prematuros, deben tener una alta sospecha de detección de disfunción renal dados los antecedentes expuestos. Si bien el antecedente de prematurez o BPN puede ser fácilmente obtenido de su registro clínico, muchas veces los eventos de daño renal agudo durante el período neonatal son escasamente documentados ${ }^{1}$. Como recomendaciones generales para preservar función renal en este grupo de niños están:

- Incluir historia perinatal en anamnesis a cualquier edad.

- Aconsejar que eviten potenciales nefrotóxicos (ej. antiinflamatorios no esteroidales, aminoglicósidos) y otros factores agravantes (deshidratación, infecciones urinarias).

- Mantener la PA bien controlada. Adecuar dieta sana, especialmente bajo consumo de sal.

- Promover lactancia materna.

- Reducir otros factores de riesgo de enfermedad renal crónica, incluyendo obesidad, diabetes y dislipidemia.

- Para los potenciales donantes vivos de riñón, cautela porque tienen mayor susceptibilidad de disfunción renal en el riñón remanente.

En forma más dirigida, el seguimiento clínico debe estructurarse de acuerdo a mayor o menor riesgo de desarrollar una enfermedad renal crónica a futuro (figura 2). Idealmente, esto debiera estar inserto en un programa específico, con la participación de pediatras y nefrólogos pediátricos con distintos grados de intervención según riesgo asignado. De no contar con el recurso del subespecialista, se debe definir la derivación a 


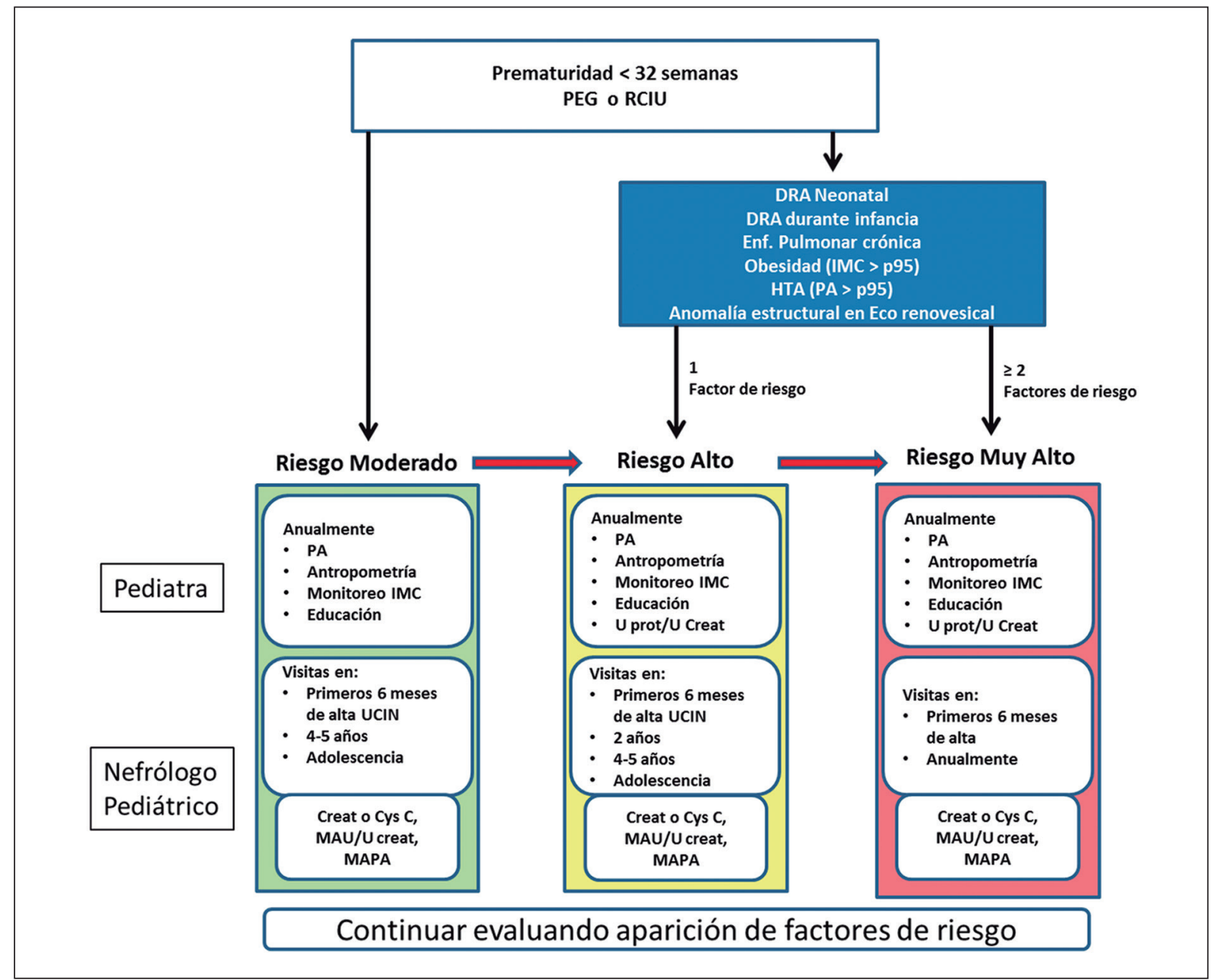

Figura 2. Algoritmo de seguimiento clínico de niños con bajo peso de nacimiento. Cys C: cistatina C; Creat: creatininemia; DRA: daño renal agudo; HTA: hipertensión arterial; IMC: índice de masa corporal; MAPA: monitoreo ambulatorio de presión arterial; MAU: microalbuminuria; PA: presión arterial; PEG: pequeño para edad gestacional; RCIU: retraso de crecimiento intrauterino; UCIN: unidad de cuidados intensivos neonatales; Ucreat: creatininuria; Uprot: proteinuria.

éste en casos de PA mayor a percentil 95 en forma sostenida, presencia de microalbuminuria o proteinuria patológica, elevación de biomarcadores de disfunción renal (ej. creatinina), y ecografía renal y vesical alterada. Para aquellos niños mayores de 5 años, en que las cifras de PA pueden estar influenciadas por el stress de la consulta médica (HTA de delantal blanco) se recomienda uso de MAPA.

\section{Conclusiones}

El enfrentamiento clínico de niños que han nacido en forma prematura requiere de un conocimiento del desarrollo anátomo-fisiológico del niño por nacer, y de las consecuencias posibles a largo plazo cuando ese proceso madurativo se afecta, se acelera o no se completa. En el caso del riñón, la prematuridad y el BPN, especialmente cuando el período neonatal presenta pa- tologías serias, se asocia a cifras elevadas de PA y a riesgo aumentado de enfermedad renal crónica en la etapa adulta, además de una progresión más acelerada o severa en algunas patologías renales no relacionadas con la prematurez. Este riesgo parece ser amplificado si se le suma una restricción del crecimiento intrauterino. Información más concreta en este tema vendrá cuando se realicen estudios en adultos mayores nacidos en forma prematura. En este contexto, es perentorio hacer un seguimiento cercano y prolongado, tanto clínico como de laboratorio, en aquellos niños nacidos antes de término, especialmente en aquellos niños muy prematuros ( $<32$ semanas EG), de muy BPN $(<1.500 \mathrm{~g})$ o con factores de riesgo renal asociado.

\section{Conflicto de intereses}

El autor declara no tener conflicto de intereses. 


\section{Referencias}

1. Luyckx V. Preterm birth and its impact on renal health. Semin Nephrol 2017;37: 311-9.

2. Ortega P, Zamora I. Peso al nacer y su repercusión nefrológica. NefroPlus 2011;4:1-10.

3. Mendoza LA, Claros D, Mendoza LI, Arias M, Peñaranda C. Epidemiología de la prematuridad, sus determinantes y prevención del parto prematuro. Rev Chil Obstet Ginecol 2016;81:330-42.

4. Chehade H, Simeoni U, Guignard JP, Boubred F. Preterm birth: long term cardiovascular and renal consequences. Current Pediatric Review 2018;14:219-26.

5. Nuyt A, Lavole JC, Mohamed I, Paquette $\mathrm{K}$, Luu T. Adult consequences of extremely preterm birth. Cardiovascular and metabolic diseases risk factors, mechanism, and prevention avenues. Clin Perinatol 2017;44:315-32.

6. Harrison, M, Golbus M, Filly R, et al. Management of the fetus with congenital hydronephrosis. J Ped Surg 1982;17:72842.

7. Gubhaju L, Sutherland M, Black MJ. Preterm birth and the kidney: implications for long-term renal health. Reprod Sci 2015;18:322-33.

8. Abitbol C, DeFreitas M, Strauss J. Assesment of kidney function in preterm infants: lifelong implications. Pediatr Nephrol 2016;31:2213-22.

9. Starr M, Hingorani S. Prematurity and future kidney health: the growing risk of chronic kidney disease. Curr Opin Pediatr 2018;30:228-35.

10. Rodríguez M, Gómez A, Abitbol C, Chandar J, Duara S, Zilleruelo G. Histomorphometric analysis of postnatal glomerulogenesis in extremely preterm infants. Pediatr Dev Pathol 2004;7:17-25.

11. Sutherland M, Gubhaju L, Moore L, et al. Accelerated maturation and abnormal morphology in the preterm neonatal kidney. J Am Soc Nephrol 2011;22:136574.

12. Kandasamy Y, Rudd D, Smith R, Lumbers E, Wright I. Extra uterine development of preterm kidneys. Pediatr Nephrol 2018;33:1007-12.

13. The low birth weight and nephron number working group. The impact of kidney development on the life course: a consensus document action. Nephron 2017;136:3-49.

14. Stritzke A, Thomas S, Amin H, Fusch C, Lodha A. Renal consequences of preterm birth. Mol Cell Pediatr 2017; 4:2. doi: 10.1186/s40348-016-0068-0.

15. Bertagnolli M, Mai Luu T, Lewandowski A, Leeson P, Nuyt A. Preterm birth and hypertension: Is there a link? Curr Hypertens Rep 2106;18:28. doi: 10.1007/ s11906-016-0637-6.

16. Hovi P, Vohr B, Ment L, et al. Blood pressure in young adults born at very low birth weight. Hypertension 2016;68:880-7.

17. South A, Nixon P, Chappell M, et al. Renal function and blood pressure are altered in adolescents born preterm. Pediatr Nephrol 2018;34:137-44.

18. Solís A, Cerda J, González C. Monitorización ambulatoria de presión arterial en escolares con antecedentes de prematurez extrema. Rev Chil Pediatr 2018;89:18-23.

19. Parkinson JR, Hyde M, Gale C, Santhakumaran S, Mosi N. Preterm birth and metabolic syndrome in adult life: a systematic review and meta-analysis. Pediatrics 2013;131:e1240-e1263.

20. Juonala M, Cheung M, Sabin M, et al. Effect of birth weight on life-course blood pressure levels among childrenborn premature: the Cardiovascular Risk in Young Finnis Study. J Hypertens 2015;33:1542-8.

21. Sundstrom J, Arima H, Jackson R, et al.
Effects of blood pressure reduction in mild hypertension: a systematic review and meta-analysis. Ann Intern Med 2015;162:184-91.

22. Boivin A, Luo ZC, Audibert F, et al. Pregnancy complications among women born preterm. CMAJ 2012;184:1777-84.

23. Davis E, Lazdam M, Lewandowski A, Worton S, et al. Cardiovascular risk in children and young adults born to preeclamptic pregnancies: a systematic review. Pediatrics 2012;129:e1552-61.

24. Liu Y, Liang M. Functional role of epigenetic regulation in the development of prenatal programmed hypertension. Kidney Int 2019;96:8-12.

25. Brenner BM, Chertow GM. Congenital oligonephropathy: an inborn cause of adult hypertension and progressive renal injury? Curr Opin Nephrol Hypertens 1993;2:691-5.

26. White SL, Perkovic V, Cass A, et al. Is low birth weight an antecedent of CKD in later life? A systematic review of observational studies. Am J Kidney Dis 2009;54:248-61.

27. Vikse B, IrgensL, Leivestad T, et al. Low birth weight increases risk for endstage renal disease. J Am Soc Nephrol 2008;19:151-7.

28. Hirano D, Ishikura $\mathrm{K}$, Uemura O, et al. Association between low birth weight and childhood onset chronic kidney disease and the National Vital Statistics Report. Nephrol Dial Transplant 2016;31:2119-26.

29. Abitbol C, Rodríguez M. The long-term renal and cardiovascular consequences of prematurity. Nat Rev Nephrol 2012;8:265-74.

30. Conti G, De Vivo D, Fede C, et al. Low birth weight is a conditioning factor for podocyte alteration and steroid dependence in children with nephrotic syndrome. Journal of Nephrology 2018;31:411-5. 